\title{
Simultaneous Measurement of Pole Figures by X-Ray Diffraction using a $2 \theta$-Position Sensitive Detector
}

\author{
L. WCISLAK and H.J. BUNGE \\ Department of Physical Metallurgy, TU Clausthal \\ Großer Bruch 23, D-3392 Clausthal-Zellerfeld, FRG
}

Pole figure measurement is usually carried out with texture goniometers equipped with a single detector. Thereby defocalization due to the sample tilt is taken into account by using broad enough receiving slits possibly with a defocalization correction. This method does not work in line-rich diffraction spectra with profuse peak overlap, particularly at high tilt angles. In this case the whole diffraction spectrum is needed for each pole figure point $\{\alpha, \beta\}$. The Integrated intensities are then to be obtained by a deconvolution procedure. Because of the necessary measuring time this is virtually impossible with a single detector. Using a position sensitive detector complete diffraction spectra can, however, be obtained for each pole figure point in reasonable times. In the present case, a $7^{0}$ linear position sensitive detector was used. The line broadening as a function of the tilt angle was measured, coordinate transformation formulae for the back-reflexion case are given and it was shown that the so obtained results exhibit smaller experimental errors than the conventional single detector measurements.

\section{INTRODUCTION}

Texture analysis is usually carried out by pole figures measurement followed by pole figure inversion i.e. the calculation of the orientation distribution function ODF (Bunge, 1982). The accuracy of the obtained ODF is the better the higher the number of input pole figures and the smaller their experimental errors. Pole figures are mainly being measured with $\mathrm{X}$-ray texture goniometers using flat samples and they are often restricted to the back-reflexion range. Pole figure inversion is then based on "incomplete" pole figures. Conventional texture goniometers are equipped with a single detector, e.g. a scintillation counter, the receiving slits of which are chosen in such a way that the total integrated intensity of the chosen Bragg-peak is registered (Bunge, 1986). This requires rather broad slits in order not to cut off rand 
parts of the Bragg peak especially at higher sample tilt angles $\chi$. This method works quite well with materials the diffraction spectra of which contain only a low number of well-separated peaks. This is the case, for example, in the basic cubic metals. If the number of diffraction peaks is higher, however, and their angular distance is smaller, then peak overlap may occur even at low or moderate tilt angles $\chi$. In some cases it is then still possible to use this method with smaller receiving slits, taking into account that part of the broadened peak may be cut off at higher tilt angles. This error is then being corrected with the help of a defocalization correction which can be determined in a random sample. But even this method has its limitations in more complex diffraction spectra, e.g. in ceramics or geological materials. In these cases it is necessary to measure the complete diffraction spectrum for each pole figure point $\{\alpha, \beta\}$ and to apply a deconvolution procedure in order to separate the increasing peak overlap with increasing tilt angle. This has to be done with small receiving slits, thus increasing the measuring time considerably. At the same time, these materials often show also lower diffraction intensities and because of lower crystal symmetry the number of required pole figures is higher. The necessary measuring times would thus be prohibitively high.

This situation can be improved by using a position sensitive detector / PSD/ which measures the diffracted intensities $\mathrm{I}(\theta)$ in a whole $2 \theta$-range simultaneously. This method has been first applied to textures analysis in the case of neutron diffraction (Bunge et al., 1982). In neutron diffraction the sample is small compared with the dimensions of the diffractometer so that Bragg-Brent.nu, focalization does not play an essential role. A curved PSD covering a $2 \theta$-rallpu of $80^{\circ}$ could thus be used without defocalization problems. In X-ray diffractiun using a flat sample in the back-reflexion technique, however, violation of the Jirgg-Brentano condition becomes the more serious the larger the $2 \theta$-range of curved PSD is. This was first treated by (Heizmann and Laruelle, 1986).

In the present investigation we used a smaller linear PSD covering $\Delta 2 \theta=7.0^{\circ}$ only. In this case the violation of the Bragg-Brentano condition is rather small. On the other hand, however, the simultaneously covered $2 \theta$-range is usually too small to measure all required pole figures at the sume time. It is then necessary to move the detector into several angular positions. This can be realized either stepwise or continuously.

If pole figures are being measured with a PSD the diffruction vectors of simultaneously measured reflexions are parallel to different sample directions. Hence, in a fixed sample position, different pole figure points $\{a, \beta\}$ are thus measured in the different pole figures. This is not a serious problem which can easily be treated by an appropriate coordinate transformation from the angles $\{\lambda, \varphi\}$ of the Eulerian cradle to the pole figure angles $\{\alpha, \beta\}$. Nevertheless, the usual equal angular scan $\Delta \chi, \Delta \varphi$ leads then to an equal angular scan $\Delta \alpha, \Delta \beta$ only in one of the pole figures. Hence, new scanning modes have to be applied in PSD texture measurement. With the small linear PSD used in the present work also this transformation is much smaller than in the case of a large curved PSD. 
Hence, the small linear PSD seems to be a good compromise between reduction of measuring time on the one hand and increasing defocalization effects on the other.

\section{THE X-RAY DIFFRACTION ASSEMBLY}

The hardware system for the X-ray diffraction measurements used in this project, shown schematicly in Fig.1, consists in principle of two main functional units. A measuring unit contains the SIEMENS X-Ray Texture Diffractometer D500/TX equipped with the DACO-MP/TX microprocessor and a controlling-analysing unit based on the DIGITAL MicroVAX II multitask, multifunctional host computer with necessary peripheral equipment.

The X-ray diffractometer contains a radiation block wherein a copper tube anode at the voltage of $40 \mathrm{kV}$ with a nickel filter emits a characteristic $K_{\alpha}$ wavelength of $\lambda=1.54056 \cdot A$, a texture goniometer with a Eulerian cradle carrying a sample to be investigated and a detection block including a position sensitive detector together with the required electronics and a gas controlling unit. The texture goniometer used for these measurements allows a precise driving and supervising of all four goniometer circles. The horizontal circles of the diffractometer namely the $2 \theta$-drive and the $\omega$-drive are equipped with step motors allowing a driving accuracy of $0.001^{0}$ in the single step. The position sensitive detector is mounted on the $2 \theta$-arm together with the $K_{\beta}$ filter system. The Eulerian cradle is mounted on the $\omega$-circle of the diffractometer. The cradle provides two additional rotatlins of the sample about the angles $\chi$ and $\varphi$. Both these $\chi$ and $\varphi$-drives are also $\mathrm{hlel}$ motor operated with the same driving accuracy namely $0.001^{\circ}$ in the single st.ej). Geometrical parameters are following, the radius of the diffractometer as well as the cradle radius are 23.5 $\mathrm{cm}$. Available diffraction angles are in the range $0^{\circ} \leq 2 \theta \leq 100^{\circ}$ limited by the own width of the cradle. The angles $\chi$ and $\varphi$ remain unlimited. The maximal scanning speed of the $2 \theta$-drive is $120^{\circ} / \mathrm{min}$ which is used when the position sensitive detector works in driving mode. Movements of several goniometer circles can be organized in any optional sequence, as a driving forwards and/or backwards, step or continuous scan, as an oscillation at certain angle ranges and frequency or as a stationary location. This enables an accomplishment of diflerent measuring methods with various scanning procedures and data analysis ways.

The position sensitive detector / PSD/ used in this experiment is a location sensitive, proportional, overflow, overpressure resistance wire electrode ionizing chamber with beryllium-entrance window. The geometrical sizes of its casing are $100 \times 50 \times$ $50 \mathrm{~mm}$. The sizes of the radiation entrance window are as follows; the linear width is $50 \mathrm{~mm}$ what corresponds to the stationary detector view angle of $7.6^{\circ}$ on the $2 \theta$-circle, the complete detection homogeneity range is, however, only ensured to the interval of $\Delta 2 \theta=7.0^{\circ}$. The height of the detector window is $10 \mathrm{~mm}$ which influences the angular resolving power. The gas control unit takes over the gas pressure control and the flow regulation of the carrier gas ( $90 \%$ argon $+10 \%$ methane). At 
the optimal operating conditions, i.e. gas pressure 7.0 bar, gas consumption $0.21 / \mathrm{h}$ and detector voltage $3.4 \mathrm{kV}$, the following detection parameters are reached. The linear location resolution is better than $100 \mu \mathrm{m}$ what corresponds to $0.01^{0}$ on the $2 \theta$-circle as the maximal angular resolving power. There are, however, possibilities to work at a weaker resolution, for example $0.1^{0}$ as it is usually chosen in the case of pole figure measurements. The detection quantum yield for the $\mathrm{Cu} K_{\alpha}$-line is about $50 \%$, this means that the exposition time has to be increased comparing to scintilation detector. The detection homogeneity fault of the PSD detector is less than $0.5 \%$ over the entire electrode length and the detection linearity assumed as a channel-to-peak allocation is 1 channel onto $0.01^{\circ}$.

The PSD-detector is followed by a multichannel analysing system / MCA/ with energy discrimination stage, location discrimination stage, multichannel analyser and an automatic step counter relating the stepmotors run to the angular detector displacement and counts channelizing in the MCA system. The used MCA version is characterized by 8192 channels, counts capacity per channel is $2 \cdot 10^{9}$ and maximal access frequency is $400 \mathrm{kHz}$. Both the texture goniometer and the multichannel analyser are equipped with their own microprocessors, so the simultaneous goniometer driving and multichannel data processing with on-line data presentation are only then possible when a multifunctional fast computer is employed.

\section{SIMULTANEOUS SCANNING METHOD IN BACK- REFLEXION TECHNIQUE}

It was already mentioned above that the simultaneous uIc: with a position sensitive detector requires a modified scanning technique compared to that used in the sequential measuring method. Conventionally, pole figures are measured one by one with the diffraction condition $\theta_{i}: 2 \theta_{i}$ being always fulfilled. The detector $2 \theta$-location corresponds to the peak profile maximum and the diffraction vector (the bisectrix between the incident and reflected beam) remains each time parallel to the sample normal vector. The mathematical algorithm describing the coordinates transformation from goniometer angles $\{\lambda, \varphi\}$ to pole figure angles $\{\alpha, \beta\}$ was first given by (Bunge et al., 1982) for neutron diffraction using spherical samples and transmission technique. In X-ray diffraction the back-reflexion technique is being used which requires an adaptation of this method. The mounting of a flat sample on the sample holder of the Eulerian cradle for the back-reflexion technique is depicted in Fig.2. Fig.3 and 4 illustrate the geometrical conditions of the simultaneous pole figures analysis. The angle $\omega$ of the sample is chosen in such a way that the diffraction vector of the middle peak $2 \theta_{i}$ is perpendicular to the sample surface. For all other diffraction angles this is not the case. The sample is then scanned through the angles $\chi$ and $\varphi$ as is shown in Fig.3. The pole figures are represented in terms of the polar coordinates $\{\alpha, \beta\}$ of the diffraction vector with respect to the sample coordinate system. These angles $\{\alpha, \beta\}$ correspond directly 
to the scanning angles $\{\chi, \varphi\}$ only for the middle angle $2 \theta_{i}$. For all other $\theta$-angles different from $\theta_{i}=\omega$ it appears an offset of the diffraction vector $|\theta-\omega|>0^{0}$. Then each new sample positioning $\{\chi, \varphi\}$ has to be appropriately transformed into pole figure points $\{\alpha, \beta\}_{(h k l)}$ as follows;

$$
\{\alpha, \beta\}_{(h k l)}=f(\{\chi, \varphi\}, \omega-\theta)
$$

We consider two cases $\theta<\theta_{i}$ and $\theta>\theta_{i}$; where $\theta_{i}=\boldsymbol{\omega}$.

In the case $\theta<\theta_{i}$ (designated by $\theta_{L}$ in Figure 3 ) it is

$$
\begin{gathered}
\alpha=\arccos \frac{\cos \chi}{\cos \left(\omega-\theta_{L}\right)+\sin ^{2}\left(\omega-\theta_{L}\right)} \\
\beta=\arcsin \frac{\sin \left(\omega-\theta_{L}\right)}{\sin \alpha}+\varphi
\end{gathered}
$$

In the case $\theta>\theta_{i}$ (designated by $\theta_{R}$ in Figure 3 ) it is

$$
\begin{gathered}
\alpha=\arccos \frac{\cos \chi}{\cos \left(\theta_{R}-\omega\right)+\sin ^{2}\left(\theta_{R}-\omega\right)} \\
\beta=360.0^{\circ}-\arcsin \frac{\sin \left(\theta_{R}-\omega\right)}{\sin \alpha}+\varphi
\end{gathered}
$$

It is to be mentioned that with the used PSD version the transformed values $\{\alpha, \beta\}$ were always very near to $\{\chi, \varphi\}$. With an angular detector range of $7^{\circ}$ in $2 \theta$ circle, the maximum deviation of $2 \theta$ from $2 \theta_{i}$ is $\pm 3.5^{\circ}$ and hence, the maximum deviation of the diffraction vector from its middle position is only $\pm 1.75^{\circ}$. The carried out computer simulation showed that $|\alpha-\chi| \leq 0.1^{0}$ which can be neglected compared with the scanning step $\Delta \alpha=5^{0}$. The deviation in $\beta$-coordinate was soinewhat larger. It was found that in an extreme situation i.e. at maximal sample till $\chi=75^{\circ}$ and maximal diffraction vector offset $\left|\theta-\theta_{i}\right|=1.75^{\circ}$, the rotation deviation $|\beta-\varphi| \leq 3.2^{\circ}$ which is comparable with the used scanning step $\Delta \beta=3.6^{0}$.

The transformation formulae were checked experimentally using silver single crystal which was cut parallel to the (001) plane. The (111) pole figure was measured in the middle position of diffraction vector so that $2 \theta_{(111)}=2 \theta_{i}$. The pole figure 
is shown in Fig.5. The same pole figure was measured again with $2^{\mathbf{0}}$-offset of the $2 \theta_{111}$ reflex from $2 \theta_{i}$ position. Plotting directly the Eulerian cradle angles $\chi, \varphi$ without coordinates correction gives the pole figure Fig.6. One sees that the (111) poles are broadened, shifted and lost their symmetries. Then the transformation $\{\chi, \varphi\} \rightarrow\{\alpha, \beta\}$ was carried out. The so obtained pole figure was practically indistinguishable from that of Fig. 5 and its maxima were repositioned identically as for the pattern pole figure from the middle position.

\section{POLE FIGURES SCANNING MODES}

If the required $2 \theta$-range is not larger than the viewing angle of the PSD i.e. $7^{0}$ in our case, then the whole data collection can be carried out in the stationary detector mode. The middle of the detector is fixed in the required $2 \theta$-position. The Eulerian cradle angles $\{\chi, \varphi\}$ are then step-scanned in equiangular intervals e.g. $\Delta \chi=5^{0}, \Delta \varphi=3.6^{\circ}$. The pole figures angles $\{\alpha, \beta\}_{(h k l)}$ are then obtained with the transformation formulae (2)-(5).

If the required $2 \theta$-range is larger than $7^{\circ}$ then the whole $2 \theta$-range has to be composed of several $7^{0}$-intervals. Since the transformation formulae (2)-(5) contain the detector middle position $\omega$, each measurement has to be performed in the stationary detector mode. Thereby the sequence of $\{\chi, \varphi\}$ scans and $2 \theta$-scans can be changed. It is thus possible to fix $2 \theta$ and scan $\{\chi, \varphi\}$ through all required positions, then to shill $2 \theta$ to the next position and again scan $\{\chi, \varphi\}$ and so on until the whole required 2 .1 ringe is covered. It is, however, equally possible to fix at first $\{\chi, \varphi\}$ and steps'inl $2 \theta$ in $7^{0}$-intervals then to go to the next $\{\chi, \varphi\}$ position and again step-scan 20-area. After the whole measurement is finished the two results are identical. Both these variants can be executed with the goniometer controlling program.

]'ole figure measurement is, however, also possible in the "moving detector mode". Thereby the detector moves continuously through the required $2 \theta$-range and the sample together with the Eulerian cradle moves in the coupled regime on the $\omega$-circle, while the $\{\lambda, \varphi\}$ position remains fixed. In this case the PSD controlling software automatically considers the actual middle position of the PSD assigning a location within the detector to a particular channel of the MCA. This procedure is the same as is used in fast PSD powder diffraction. After one run the MCA contains the whole $2 \theta$ - spectrum (larger than $7^{\circ}$ ). If this method is applied to pole figure measurement, however, one has to take into account the variable middle position $\boldsymbol{\omega}$ of the detector in equations (2)-(5). Hence, with the same $\{\chi, \varphi\}$ values variables $\{\alpha, \beta\}$ are obtained when a particular diffraction peak (hkl) moves from $2 \theta_{L}$ through $2 \theta_{i}$ to $2 \theta_{R}$, see Fig.3 and 4. Hence, the stored intensity in a particular MCA channel corresponds to an integral over a small line in the pole figure centered at $\{\alpha, \beta\}=\{\chi, \varphi\}$. As long as the length of this line is comparable with the scanning steps $\Delta \chi, \Delta \varphi$ or $\Delta \alpha, \Delta \beta$ then this integration does not reduce the accuracy of the obtained pole figure. Quite the contrary, the integration may even be advantageous since it improves the grain 
statistics during the measurement. In the "moving detector mode" the subroutine executing the transformations (2)-(5) is thus not activated.

\section{DEFOCALIZATION BROADENING}

When the sample is being tilted through the angle $\chi(\sim \alpha)$ then only one line of its surface can remain on the focussing circle. The upper part of the illuminated area is, for instance, behind it whereas the lower part is in front of it. Hence, the whole peak is no longer focussed at the detector position. The actual shape of the broadened line depends on the vertical extension of the illuminated area on the sample surface and it may even depend on the intensity distribution of the irradiating beam within this area. Besides this, the broadening also depends on the Bragg angle $\theta$. Fig. 7 exemplifies a peak broadening for different tilt angles $\chi(\sim \alpha)$. The exact form of this broadening depends on the particular choice of the primary divergence slits. This line broadening has to be taken into account when integrated peak intensities are to be determined. As long as the peaks of different reflexions (hkl) are wide enough separated one can simply integrate the intensity over the whole peak profile (i.e. sum up the counts of the corresponding channels of the MCA). It is, however, also possible to chose an increasing integration interval still covering a whole broadened peak profile with increasing tilt angle $\chi(\sim \alpha)$. This lattur method was chosen in the present case. Fig.8a shows the pole figures of the (111)-reflex of a cold rolled copper sheet, measured with the PSD in stationary li.lactor mode, integrating over the appropriate peak width $\Delta 2 \theta$ (the surface area Indiler the peak envelope) i.e. an adapted number of channels for each tilt angle $\lambda(\sim \alpha)$. The results are compared with an evaluation of the same primary data integrating over a constant $2 \theta$-interval, namely $\Delta 2 \theta=1.1^{\circ}=$ const. corresponding to the maximum receiving slit width available in a scintillation counter measurement, Fig.8b. For a usually used angular resolving power of $0.1^{\%} /$ channel for pole figure mensurement this corresponds to 11 channels of the MCA. Finally, a measurement with $\Delta 2 \theta=0.5^{\circ}=$ const. (corresponding to $5 \mathrm{MCA}$ channels) was simulated Fig. $8 \mathrm{c}$. In Figure $8 \mathrm{a}$ the whole peak profile is integrated up to the maximum tilt angle. The simulated "scintillation counter measurement" shows already "cut off" effects at high tilt angles and the simulated "5 channels" measurement depicts this effect to an even higher degree. The pole figure data of Fig. 8 were then used as input data for ODF analysis. This allows to calculate the error coefficients $\Delta C_{\lambda}$ (averaged over $\mu$ and $\nu$ ), shown in Fig.9. It is seen that the 5 channels measurement shows higher error values particularly for low $\lambda$-values which is due to systematic errors in the pole figures, i.e. the cut off effect at higher tilt angles. The error coefficients of the "scintillation counter" and "variable $\Delta 2 \theta$-interval" measurement Fig. 9 are still distinguishable. This shows that the routine measurement of pole figures with scintillation counter (without defocalization correction) gives already quite good results due to the least squares averaging of the pole figure inversion method. Nevertheless are these two 
pole figures as raw measured data still clearly distinguishable at higher tilt angles. Fig.10 illustrates difference pole figures, i.e. differences between pole figure data in the case of "variable $\Delta 2 \theta$-interval" minus " 11 channels" and "variable $\Delta 2 \theta$-interval" minus " 5 channels" respectively. All local maxima and contour levels were presented in counts/sec to avoid averaging effects which always appear in the normalization procedure. It is seen that the results are identical or similar in the center of the pole figures and that the differences increase with increasing tilt angle. Finally, Fig.11 shows the recalculated pole figures which are a least squares compromise between the errors contained in all pole figures used for the ODF calculation. It is seen that the recalculated pole figures show more details especially at the higher tilt angles.

Fig.8-11 confirm the advantage of the use of PSD measurements in texture analysis compared with the conventional single detector method. In the present example of sufficiently separated peaks the same results could of course also be attained with the single detector method. In the case of line-rich diffraction spectra with $\theta$-overlap, however, the PSD method is indispensable. A deconvolution of the broadened peak shapes according to Fig. 7 with possible $\alpha$-overlap has then to be involved in the evaluation programs.

\section{REFERENCES}

1. Bunge H.J. Textures in Material Science, (1982) Butterworths, London

2. Bunge H.J., Wenk H.R. and Pannetier J. (1982).

Neutron Diffraction Texture Analysis using a $2 \theta$-Position

Sensitive Detector. Textures and Microstructures 5, 153-170

3. Bunge H.J., Experimental Techniques of Texture Analysis, in: Experimental Techniques of Textures Analysis.

Ed. H.J. Bunge, DGM Verlag-Ges., Oberursel (1986) p. 1-29

4. Heizmann J.J. and Laruelle C. (1986)

Simultaneous Measurement of Several X-Ray Pole Figures.

in: J. Appl. Cryst. 19, 467-472 


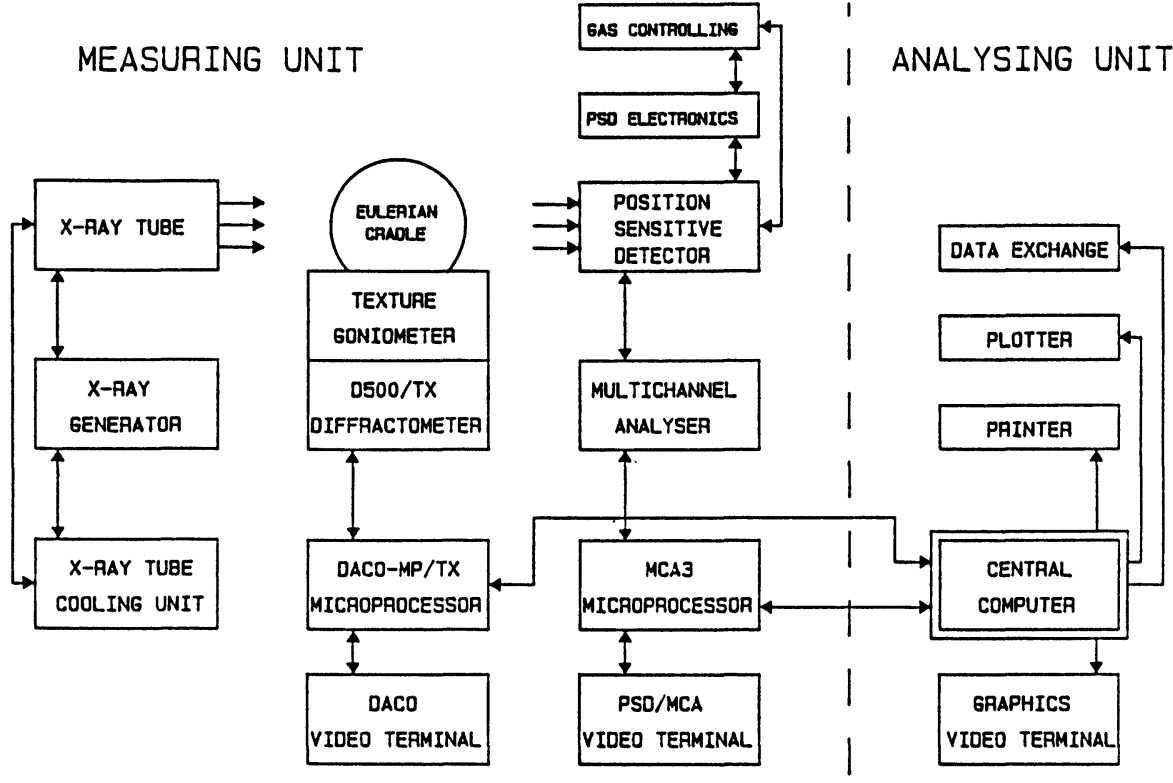

Fig.1 : Schematic diagram of experimental equipment involved in the research project

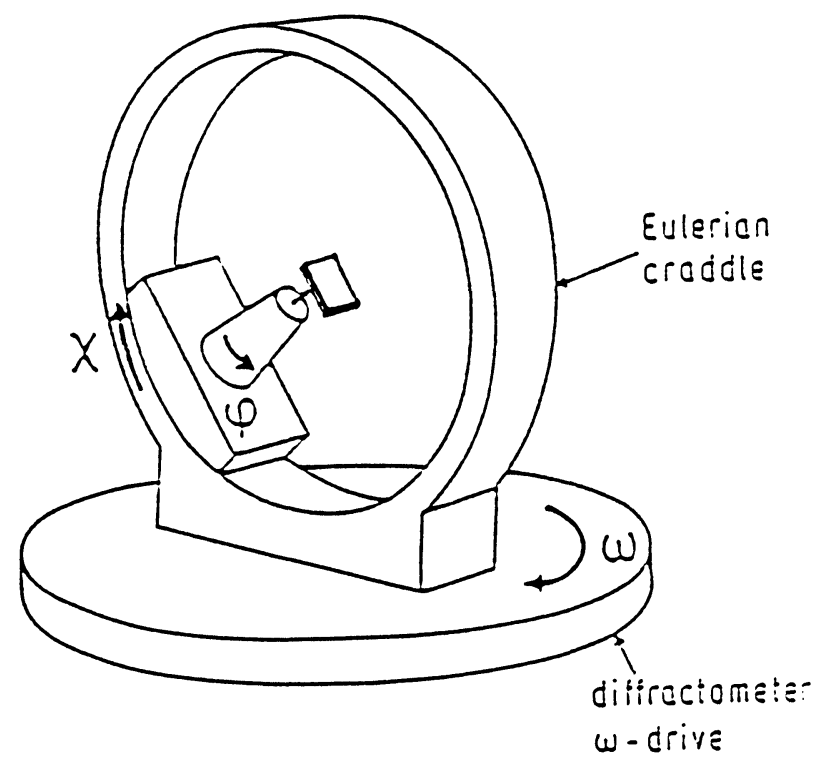

Fig.2 : Mounting way of a flat sample in the Eulerian cradle for the back-reflexion technique 


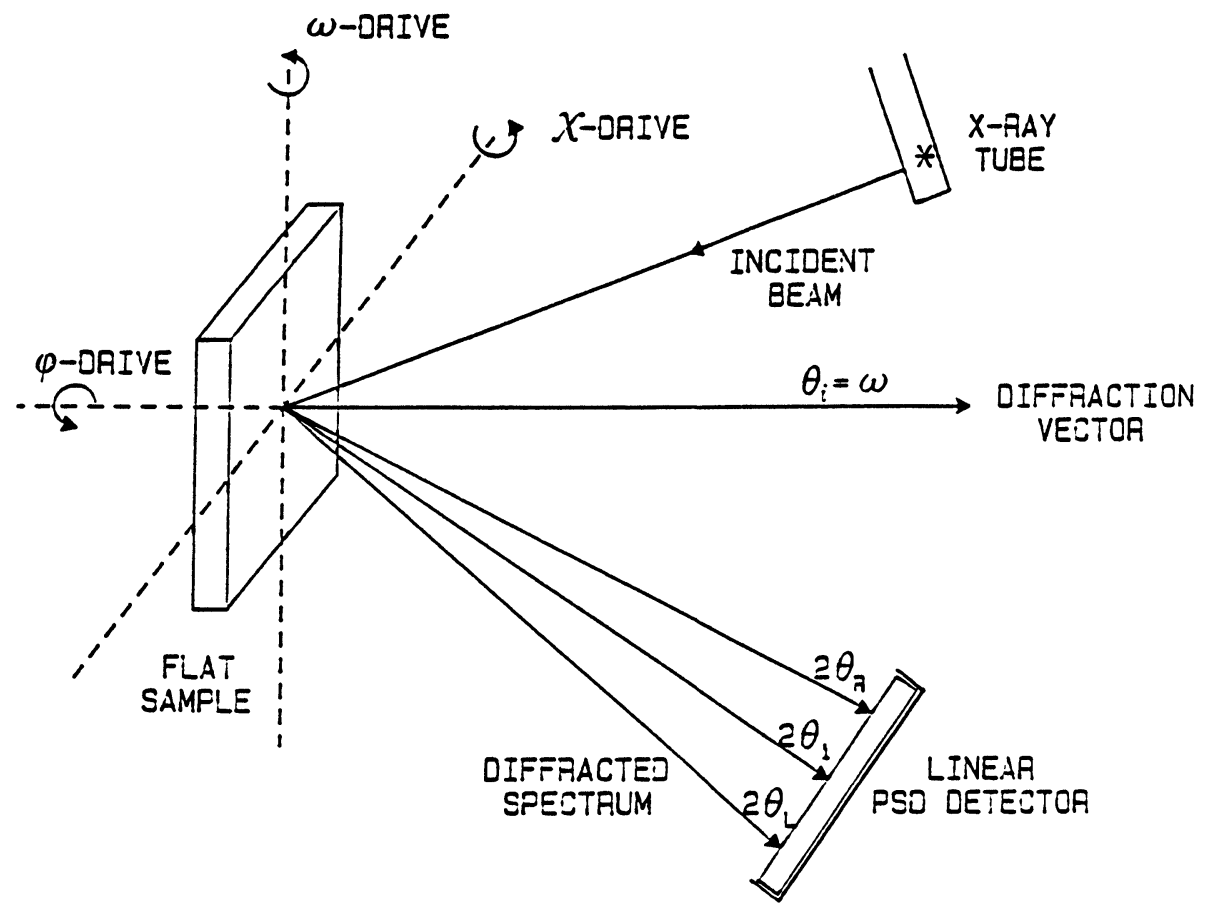

Fig.3 : Schematic view of the measurement geometry for flat samples in the back-reflexion technique

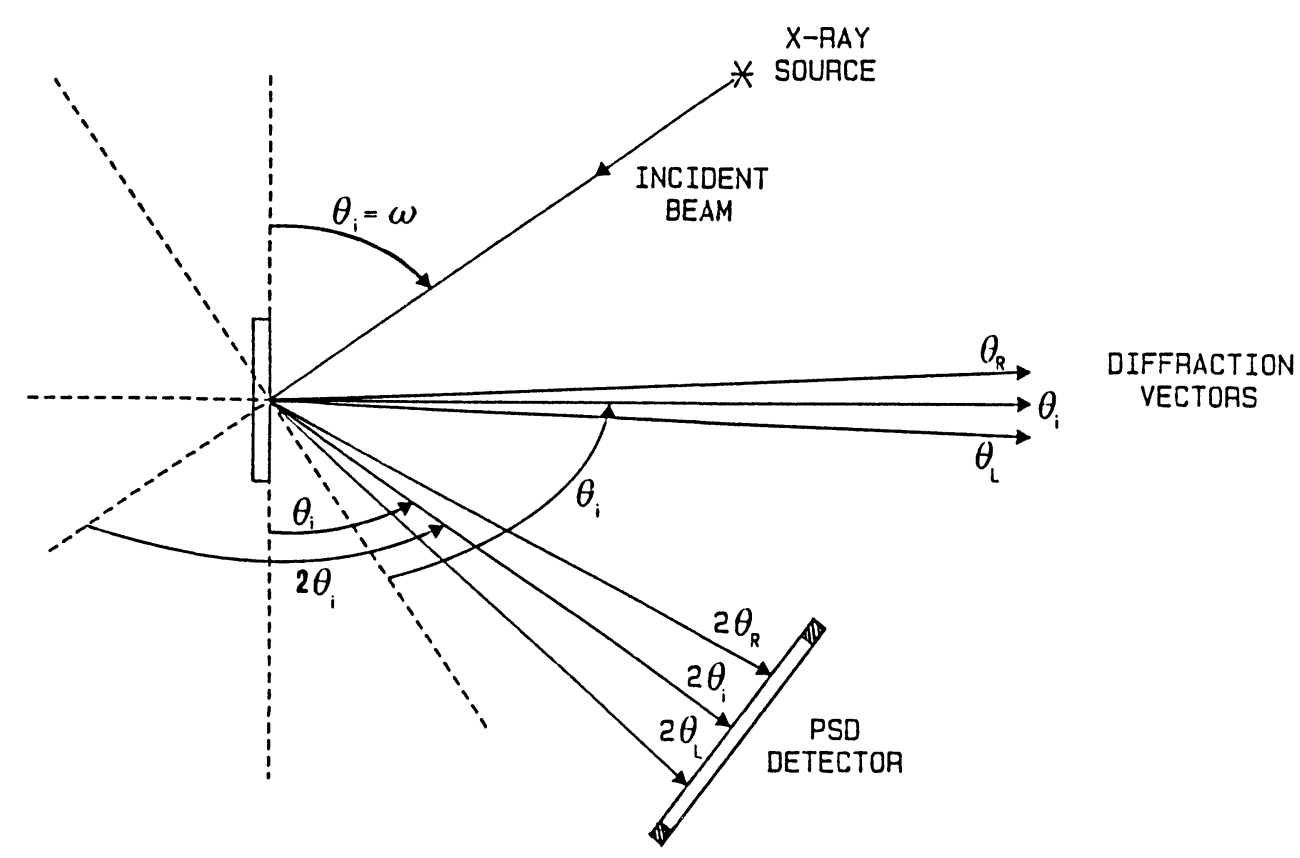

Fig.4: Geometrical conditions at a simultaneous measurement of pole figures using a $2 \theta$-position sensitive detector 


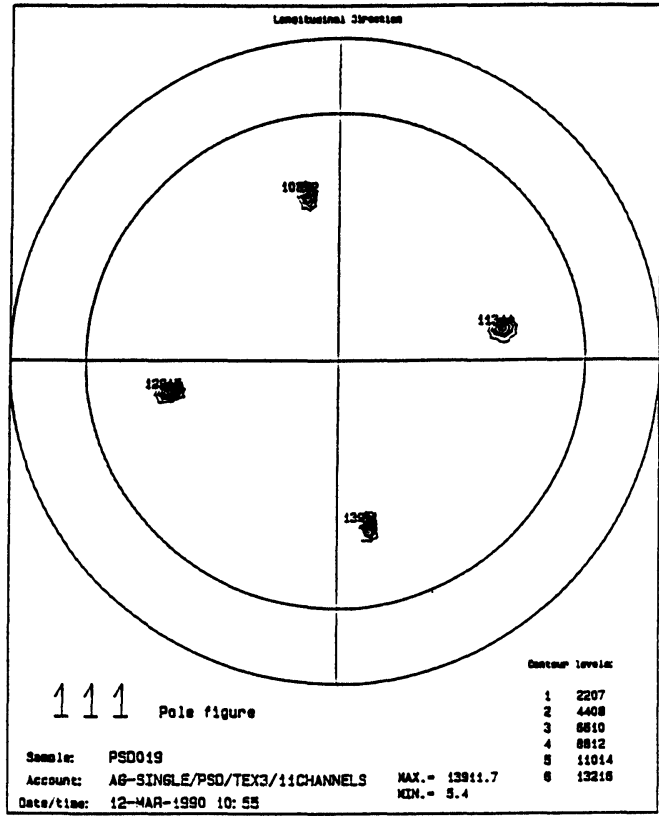

Fig.5 : Pole figure measured at the (111)-reflex for a single-crystai specimen of silver. This pole figure was measured at the fulfilled diffraction condition $\theta: 2 \theta$

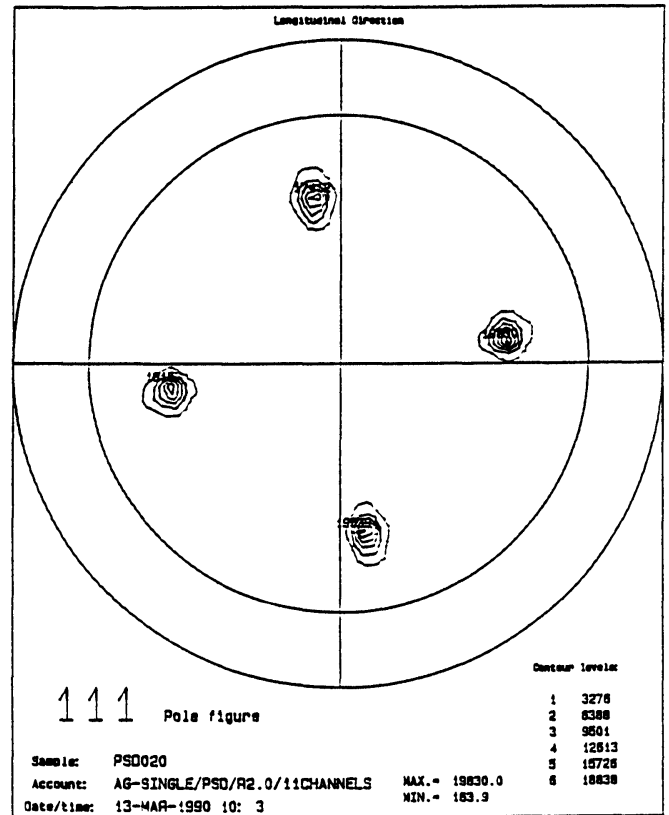

Fig.6 : Pole figure measured at the (111)-reflex for a single-crystal specimen of silver. This pole figure was measured at the $2.0^{\circ}$-offset of the (111)-reflex 


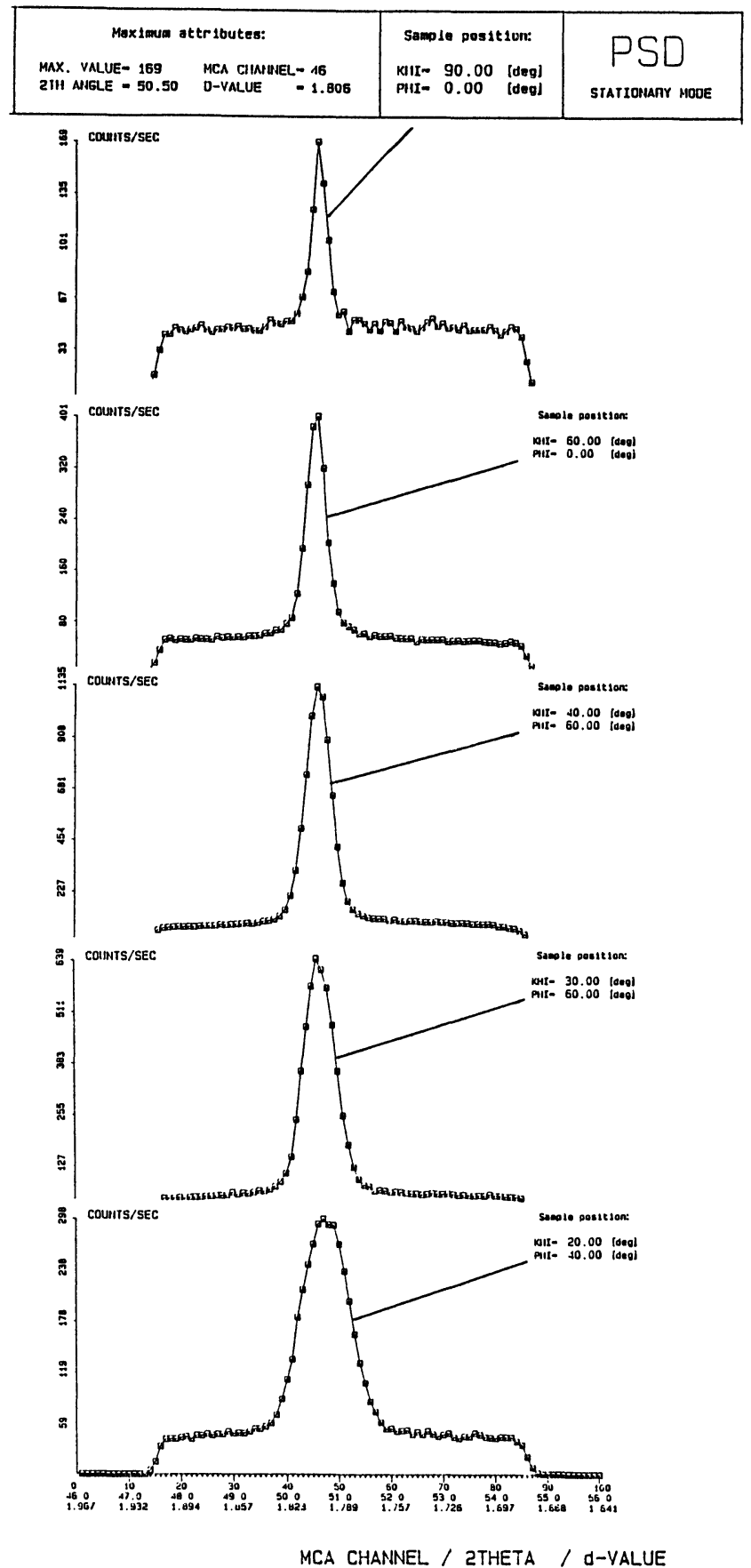

Fig. 7 : Relationship between the peak broadening and the sample tilt angle Several spectra of the (200)-reflex of cold rolled copper sheet were measured with the PSD in stationary detector mode 

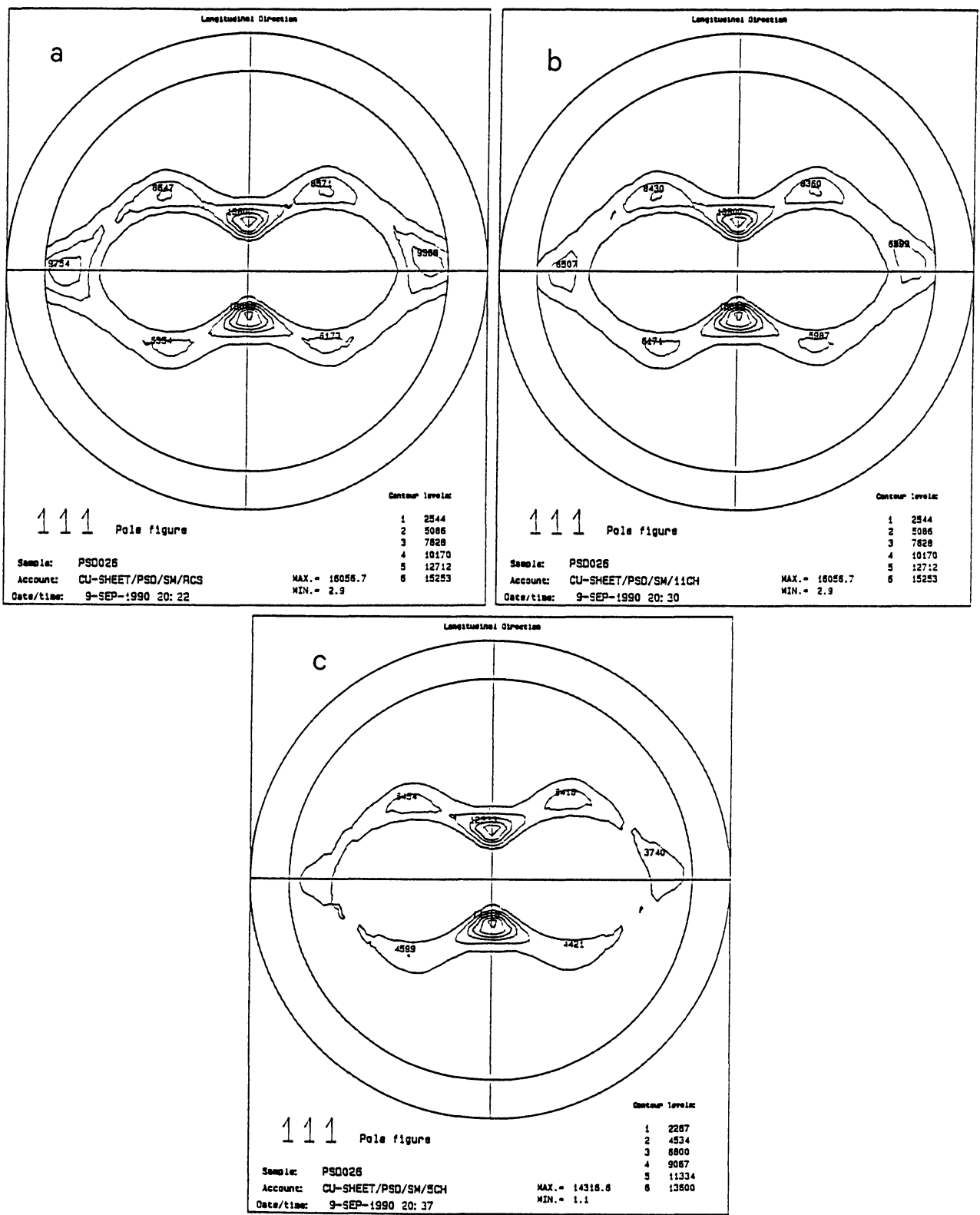

Fig.8 : Pole figure measured with the PSD in stationary mode. integrating

(a) over the required peak profile width "variable $\Delta 2 \theta$-interval",

(b) over the constant $\Delta 2 \theta$-interval "11-channels",

(c) over the constant $\Delta 2 \theta$-interval "5-channels" 


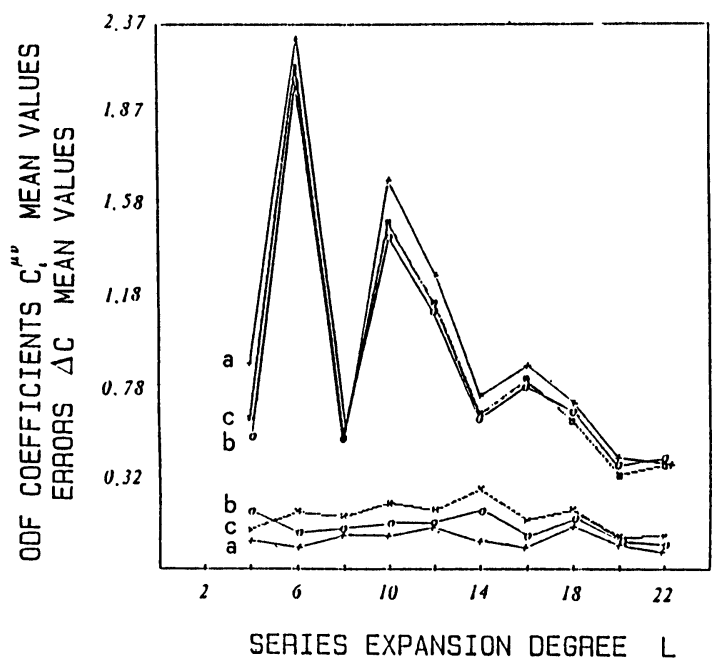

Fig.9 : Error coefficients obtained in the ODF calculation from the (111),(200) and (220)-pole figures raw data

(a) variable $\Delta 2 \theta$-interval "adapted channels summation",

(b) constant $\Delta 2 \theta$-interval "11-channels",

(c) constant $\Delta 2 \theta$-interval "5-channels"
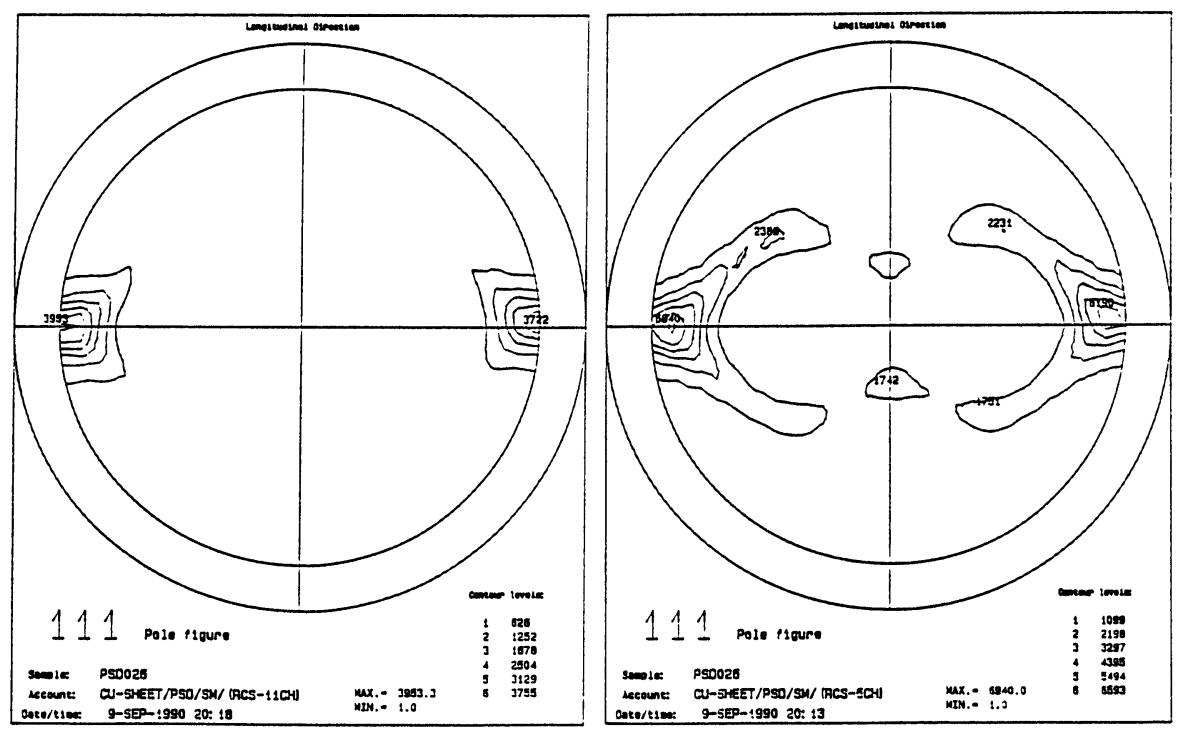

Fig.10 Difference pole figures, differencies between pole figures data for: (a) "variable $\Delta 2 \theta$-interval" minus "constant interval 11-channels", (b) "variable $\Delta 2 \theta$-interval" minus "constant interval 5 -channels" 

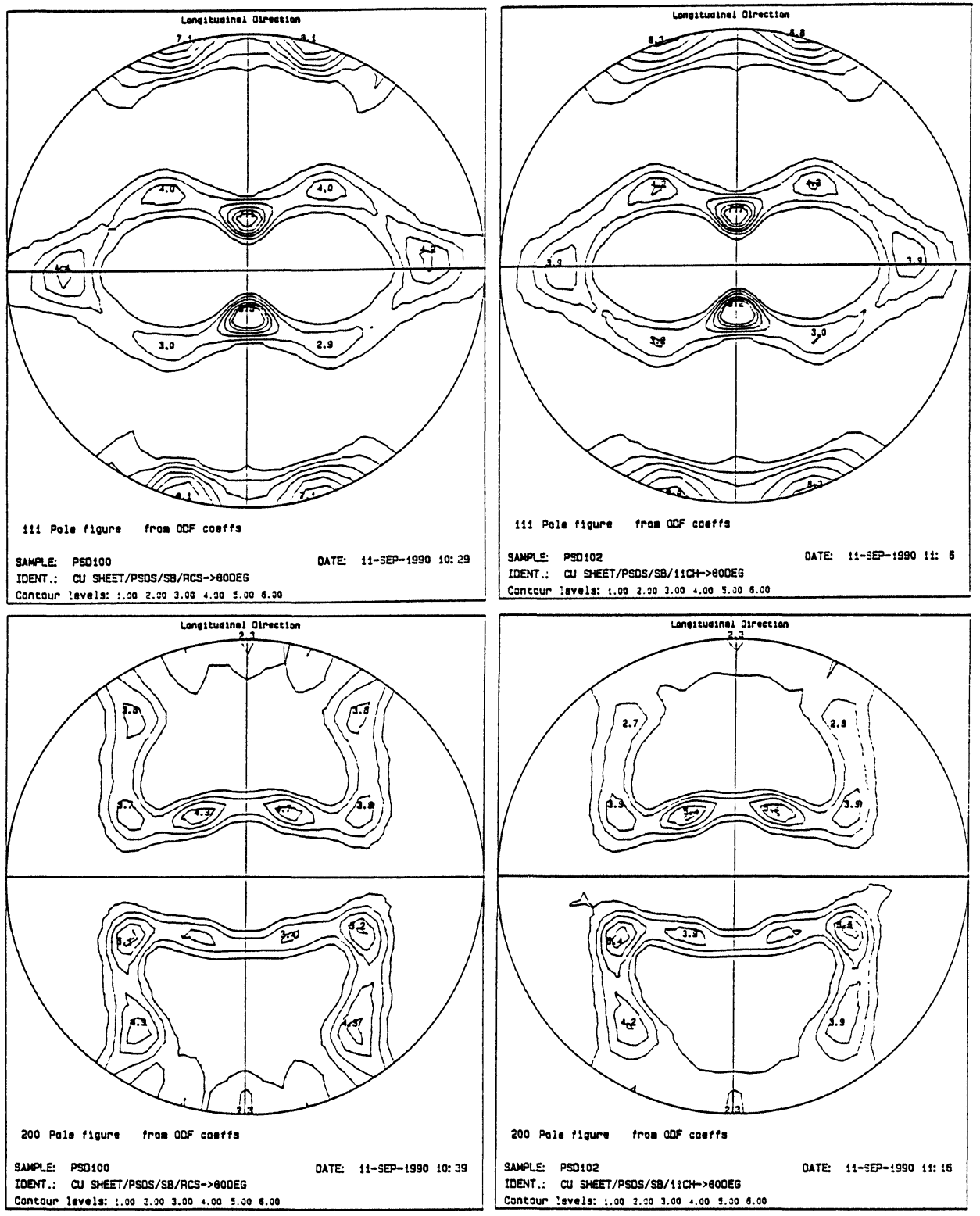

Fig.11 Recalculated pole figures in the case of ODF input data for (a) "variable $\Delta 2 \theta$-interval", (b) "constant interval 11-channels" 